\title{
The Molecular Biology of the Interactions Between Trichoderma spp., Phytopathogenic Fungi, and Plants
}

\author{
S. L. Woo, F. Scala, M. Ruocco, and M. Lorito
}

Department of Arboricoltura, Botanica e Patologia Vegetale, Plant Pathology Section, University of Naples and CNR IPP-Institute for Plant Protection, Via Università 100, 80055 Portici (Napoli), Italy.

Accepted for publication 19 June 2005.

\begin{abstract}
Woo, S. L., Scala, F., Ruocco, M., and Lorito, M. 2006. The molecular biology of the interactions between Trichoderma spp., phytopathogenic fungi, and plants. Phytopathology 96:181-185.

Trichoderma-based biofungicides are a reality in agriculture, with more than 50 formulations today available as registered products worldwide. Several strategies have been applied to identify the main genes and compounds involved in this complex, three-way cross-talk between the fungal antagonist, the plant, and microbial pathogens. Proteome and

porters, enzymes and other proteins that produce or act as novel elicitors of induced resistance, proteins responsible for a gene-for-gene avirulent interaction between Trichoderma spp. and plants, mycoparasitism-related inducers, plant proteins specifically induced by Trichoderma, etc. We have transgenically demonstrated the ability of Trichoderma spp. to transfer heterologous proteins into plant during root colonization, and have used green fluorescent protein and other markers to study the interaction in vivo and in situ between Trichoderma spp. and the fungal pathogen or the plant.
\end{abstract} genome analysis have greatly enhanced our ability to conduct holistic and genome-based functional studies. We have identified and determined the role of a variety of novel genes and gene-products, including $\mathrm{ABC}$ trans-
Additional keywords: biopesticides, proteomics, symbiosis.
Biocontrol fungi of the genus Trichoderma have developed an astonishing ability to interact, both parasitically and symbiotically, with different substrates and living organisms, including plants and other microbes $(10,12)$. These fungi can utilize a variety of nutrient sources, are among the most resistant microbes to natural and man-made chemicals and toxins, and are able to effectively degrade some of them, including hydrocarbons, chlorophenolic compounds, polysaccharides, and xenobiotic pesticides (11). Many strains of Trichoderma spp. are strong opportunistic invaders, fast growing, prolific producers of spores and powerful antibiotic producers. These properties make these fungi ecologically very successful since strains have been found in agricultural, native prairie, forest, salt marsh, and desert soils of all climatic zones (including tundra, Antarctic, and tropics), as well as in lake water, dead plant material, living roots of virtually any plant species, seeds, and air (21).

Trichoderma spp. are widely used in agriculture and industry: T. reesei strains are typically applied for protein production, and biocontrol isolates are used as biopesticides, bioprotectants, biostimulants, and biofertilizers on a wide variety of plants (10). This is possible because Trichoderma propagules can be produced cheaply and in large quantities, highly concentrated, both in liquid (water or oil) and dry (granules and wettable power) formulations, and stored for months without losing the beneficial effect for the crop (10). Today, more than 50 different Trichoderma-based agricultural products can be found on the Internet as registered in many different countries in five continents, and are sold and applied to protect and improve yield of vegetable, orna-

Corresponding author: M. Lorito; E-mail address: lorito@unina.it

DOI: 10.1094/PHYTO-96-0181

(C) 2006 The American Phytopathological Society mentals, and fruit trees. In addition, methods have been developed to genetically modify these fungi in a very precise manner, which allows the improvement of their ability to secrete desired enzymes, kill plant pathogens, or stimulate plant growth and resistance to diseases $(17,23)$. These results are based on the research carried out on Trichoderma spp. in the last 20 years that have uncovered the molecular and genetic basis of the mechanisms involved in many beneficial or useful biological processes $(10,22,29)$.

Research data accumulated in the past few years have produced a completely novel understanding of the way by which these fungi interact not only with other microbes, but especially with plants and soil components. This has opened an avenue of new applications, both in agriculture and biotechnology, that exploit the ability of these fungi to change plant metabolism and resistance to biotic and abiotic stresses (29). In fact, they can be used either to improve crop plant fitness or increase the natural ability of some plants to degrade toxic compounds in soil and waters (11). Many of these concepts have been extensively covered in recent reviews $(9,11)$ and will not be considered here. Instead, this paper will discuss some of the latest studies, including many still unpublished, on the multiple interactions that involve Trichoderma strains (mainly $T$. atroviride strain $\mathrm{P} 1$ and T. harzianum strain T22, used as models), crop plants, and soilborne fungal pathogens. The use of relatively novel tools to investigate these complex processes, such as proteomic analysis, use of gene expression reporter systems, and high throughput methods to study gene function, has demonstrated that a molecular cross-talk is established between Trichoderma spp., the plant, and the pathogen, and has permitted the identification of a variety of signaling molecules that may strongly influence the life and physiology of many crops (9). Some of these molecules may act as hormones and stimulate plant growth and development, while others func- 
tion as elicitors of disease resistance and activate many plant genes in differential ways, depending on the challenge provided by different pathogens. Therefore, the presence and the activity of these fungi in the soil actually may determine how much the plant will suffer from an environmental stress, or make de facto incompatible, an otherwise compatible interaction between the plant and a pathogenic microbe.

Proteomic analysis of Trichoderma spp. interactions. Many papers have been published in the last 2 decades that describe different factors more or less involved in the mechanism of interaction between Trichoderma spp. and other fungi, bacteria or, to a lesser extent, plants $(2,9)$. Given the proven ability of agriculturally useful Trichoderma strains to secrete enzymes and other antimicrobial compounds, a lot of attention has been focused on the role of cell wall degrading enzymes (CWDEs) and antibiotics in mycoparasitism or antagonism with phytopathogenic microbes $(6,13,18)$. Although studies based on mutants and targeted gene disruption have been carried out by different groups, the redundancy in the Trichoderma genome of CWDEencoding genes has made it difficult to identify enzymes required for biocontrol independently of the strain and the system used $(1,4,23)$. The discrepancies sometimes observed in the literature clearly suggest that these fungi have a variety of molecular weapons that can be used in different combinations depending on the microbe they are confronting or the plant they are colonizing. On the other hand, this has also demonstrated the abundance of biotechnologically valuable proteins and secondary metabolites that Trichoderma spp. can provide. Much less has been done on identifying genes and proteins that play a role in the interaction between the different Trichoderma strains and the plant. Most of the studies have been concentrated on a few plant genes that are differentially expressed when these fungi are established on the roots, often independently of the pathogen presence $(26,28)$. However, the complexity of the system, which requires more than one player to act at the same time, has strongly indicated that a more holistic approach is necessary to make a leap forward in our understanding of the biological processes involved.

For these reasons, several laboratories have recently started or planned to use proteomic (7) and functional genomic analysis in the attempt to obtain an overall picture of the changes that occur in the Trichoderma, plant, and pathogen expressomes when they "talk" to each other, especially when an increase in disease resistance is generated. For instance, the 5th and 6th frame work programs of the European Union (projects: Functional genomics and proteomics of Trichoderma antagonist strains for industry and agriculture http://www.trichoderma.org/ and Enhancement and exploitation of soil biocontrol agents for bioconstraint management in crops http://www.2E-BCAs.org) have given grants to fully investigate and exploit the genes and the molecules involved in this three-way interaction between biocontrol fungi, plants, and microbial pathogens, by using Trichoderma spp. and vegetables as model systems. Trichoderma expressed sequence tag (EST) libraries made under a large variety of inducing conditions are being processed, and entire genome sequencing initiatives have been launched both in the United States and Europe. Final results are expected to be released soon, even though it already appears that major differences exist within the genus: over $70 \%$ of the T. harzianum genes and ESTs sequenced so far, as obtained from a variety of conditions, are different from those of $T$. reesei today present in databases (22).

Most of the available data, although published only in a limited amount, concerning the changes occurring in Trichoderma spp. during these complex interactions, come from 2D gel-based separation of the proteome followed by matrix assisted laser desorption/ionization time of flight (MALDI-TOF) and in silico analysis of the differential spots. The first papers reporting data on the proteome of $T$. harzianum or T. atroviride grown on liquid substrates enriched with different carbon sources, including pathogen cell walls, have been published recently $(7,8,15)$. In our laboratory, we developed a method to separate the proteome of T. harzianum strain T22 and T. atroviride strain P1 from that of Rhizoctonia solani, Botrytis cinerea, or Pythium ultimum and that of bean or tomato plants, while all three components are grown together under controlled conditions (P. Ambrosino, R. Marra, and M. Lorito, unpublished data). A large variety of protein spots differentially expressed during the two- (Trichoderma-pathogen or Trichoderma-plant) or three- (Trichoderma-plant-pathogen) way interaction have been selected (spots identified as on, off, decreased or increased) from the proteome of each component and subjected to MALDI-TOF and database comparison for protein identification via CSI (cross species identification). We have found that the Trichoderma expressome is strongly and differentially changed when different host pathogens are detected or while the fungus is colonizing a plant root. On the other hand, the plant proteome showed more than 100 proteins that are turned on or off by the presence of Trichoderma spp. A large number of proteins involved in disease resistance processes (pathogenesis related $[\mathrm{PR}]$, signaling proteins, etc.) have been found in bean and tomato colonized by Trichoderma spp. with and without the pathogen. This has demonstrated, for instance, that several resistance genes used for recognition of microbial metabolites are upregulated or activated by the presence of the beneficial fungus and probably allow the plant to recognize it (described below).

The studies performed on the proteome of Trichoderma spp. interacting with pathogens and plants have already provided many novel data that improve our understanding on how these fungi search for the pathogen, talk to the plant, and protect themselves from toxicants. For instance, a battery of enzymes (mainly chitinases and glucanases), used to search for the cell walls of the fungal host or dead plant material in the environment and release mycoparasitism or colonization inducers sensed by Trichoderma spp., have been identified and are being studied also by targeted gene knockout $(5,23)$. These low molecular weight (MW) inducer molecules have been isolated, their structure identified and found to stimulate fungal growth, antibiotic and enzyme production, mycoparasitism, and biocontrol effect $(24,25)$. These results will be published shortly and demonstrate that the low MW inducers derived from the host fungus cell wall are made of small oligosaccharides (i.e., a monoacetylchitobiose attached to two cellobiose units) bound to an amino acid moiety (valine or ornitine). Evidence that some of these compounds are also recognized by the plant has been obtained (M. Ruocco, S. L. Woo, and M. Lorito, unpublished data). In addition, avr-like genes and novel proteins functioning as typical avirulence compounds have been found in Trichoderma spp., and are probably involved in induced resistance and recognition of the nonpathogenic fungus (described below). For the first time, a variety of ABC transporters have been found to be used by Trichoderma spp. to withstand the impact of toxins produced by other microbes (phytopathogenic or not) or the plant. In our laboratory, we have isolated four different $A B C$ transporter encoding genes more or less specifically expressed when the fungus is exposed to culture filtrates and antibiotics produced by $B$. cinerea, $P$. ultimum, and other microbial pathogens or to injured plant tissues. One of these genes has been found to be required for full resistance to such biochemical stresses by comparing the response of targeted knockout mutants and the wild type (T. atroviride strain P1). A paper containing the details of this study will be published soon (14). Interestingly, the sequences of the four $\mathrm{ABC}$ transporter genes obtained so far have a higher degree of similarity with some of their homologs from other organisms than among themselves. Therefore, we consider that the presence of a large number and variety of effective membrane pumps supports the general ability of these fungi to be strong competitors and explains the innate high level of resistance of Trichoderma spp. to an astonishing assortment of chemicals 
(over 30 pesticides, hydrocarbons, phenolics, arsenic, etc.) and natural toxins produced by other microbes (i.e., lipodepsipeptides of Pseudomonas spp.) $(6,10,11)$.

Trichoderma sp. induces systemic disease resistance by producing molecules that are "sensed" by the plant. The ability of Trichoderma spp. to systemically activate plant resistance mechanisms against fungal pathogens has been demonstrated by several studies. These fungi were able to beneficially modify the response of more than 10 different dicots and monocots, including graminaceae, solanaceae, and cucurbitaceae, to infection by fungi (R. solani, B. cinerea, Colletotrichum spp., Magnaporthe grisea, Phytophthora spp., Alternaria spp., etc.), bacteria (Xanthomonas spp., Pseudomonas syringae, etc.), and even viruses (Cucumber mosaic virus) (literature citation 9 provides a review). Most of these experiments, which have been largely performed only at a laboratory scale, demonstrate that root or foliar colonization by selected Trichoderma strains, classified as T. harzianum, T. virens, $T$. atroviride, and T. asperellum, reduces development of symptoms caused by one or two different pathogens applied separately on a site different than that of the biocontrol fungus. Observation of this phenomenon has been extended to a molecular level at least for one system, by discovering that a Trichoderma-based root treatment makes the extracts from cucumber plant tissues more antimicrobial than untreated controls. This has been found to be correlated with the upregulation of different PR and defense-related proteins (chitinases, glucanases, peroxidases and specific phytoalexins) and enzyme activities typically increased during a pathogenic attack (HPL, PAL1, etc.) $(26,27)$. It is our opinion, as recently discussed in more detail elsewhere (9), that the type of induced resistance mechanism so far demonstrated for Trichoderma spp. resembles that of the induced systemic resistance (ISR) caused by rhizobacteria (RISR). This mechanism, by which Trichoderma protects plants, is today considered of primary importance, at least in some applications, compared with the "classical" direct mycoparasitism and competition occurring at the expenses of a phytopathogenic host $(2,9)$. A growing number of experts support the hypothesis that ISR and not antagonistic ability is the mechanism behind some of the best and widespectrum performances of well-known commercial strains used all over the world as plant protectants. This concept is highly attractive since much has still to be discovered about the molecules and genes involved and the obvious similarity between the mechanisms of plant interaction with Trichoderma spp., mycorrhizal fungi, rhizobacteria, or microbial pathogens. Regardless, the actual application in agriculture of Trichoderma strains specifically selected for their ISR ability is far from being a reality, mainly because of the lack of sufficient understanding, persistence, and reproducibility of this process. The ISR effect seems to be strongly dependent on the strain-plant combination applied, as well as the growth promotion effect (topic not considered in this review) (29), which has been found to occur only with some but not all cultivars of tomato (M. Ruocco and M. Lorito, unpublished data) and maize (9).

More recently, authors have reported that Trichoderma hyphae do penetrate into the root cortex although the colonization is limited to the first few cell layers, probably because of the local reaction by the plant tissues (i.e., deposition of callose barriers) (26; A. Bolwerk, M. Lorito, B. J. J. Lugtemberg, and G. V. Bloemberg, unpublished data). The effect (expression of plant defense genes) and the occurrence of a direct interaction between the plant living cells and the fungal structures have been observed and demonstrated in vivo. However, it is still unclear the mechanism by which Trichoderma spp. provides a stimulus for the plant defense mechanism and remains an avirulent symbiont that grows together with the plant root system. In fact, many strains of Trichoderma are capable of producing a variety of powerful plant-degrading enzymes (they are common saprophytes thriving on dead plant tissues) and over 200 types of antibiotics highly toxic to cells of any macro- and microorganism (12). In addition, our data indicate that Trichoderma culture filtrates contain macromolecules and low molecular weight compounds that induce strong changes in cytosolic $\mathrm{Ca}^{2+}$ level in isolated plant cells and may cause programmed cell death (L. Navazio, B. Baldan, R. Moscatiello, A. Zuppini, S. L. Woo, P. Mariani, and M. Lorito, unpublished data). In other words, many Trichoderma strains living in agricultural soils have the intrinsic ability to be phytopathogens and probably all the necessary molecular weaponry to act as such, but instead have developed the habit of interacting beneficially with the plant by stimulating root development (thus, increasing their nutrient base out of root exudates) and plant resistance to pathogenic microbes (thus, limiting competitor growth). The mechanisms by which Trichoderma limits its penetration into the plant tissues are not yet understood, although this may be correlated with its ability to activate plant defense systems by producing avr-like proteins and other types of elicitors (described below).

A first answer to the question of how some Trichoderma strains elicit plant defenses has been recently provided by experiments performed in our laboratory in collaboration with P. de Wit at Wageningen University. We transformed the biocontrol strain P1 of $T$. atroviride with the gene encoding for the Avr4 protein of Cladosporium fulvum, both under a strong constitutive or an inducible promoter. Avr4-secreting mutants overexpressing the $C$. fulvum transgene were applied to tomato seeds of cultivars containing and not containing the corresponding resistance gene $C f 4$. Seeds from the $C f 4$ plant produced fewer stunted and less healthy plantlets than the non-Cf4-containing cultivar, which was similar to the untreated controls. If the Trichoderma avr mutants were applied on the roots of mature $C f 4$ plants, the rapid appearance of many hypersensitive response (HR)-resembling necrotic zones was observed on root and foliar surface (M. Ruocco and M. Lorito, unpublished data). All together, the data collected demonstrate that a molecular cross-talk is established between Trichoderma spp. and the colonized plant, and that the fungus is able to transfer to receptive crops molecules that are recognized and able to activate a variety of processes such as defense against biotic offenders (microbes, insect, nematodes, and viruses) and accelerated development or resistance against abiotic stresses (i.e., drought and nitrogen deficiency). Moreover, this opens the possibility of using selected Trichoderma strains to transfer into plants useful molecules, given the availability of promoters constitutively expressed or activated by the interaction with the plant (19) or the pathogen $(20,30)$.

If Trichoderma sp. is able to exchange molecular signals with the plant, what are these highly effective compounds that can dramatically change the plant proteome and stress the resistance status? Our experiments with fungal $a v r$ genes coupled with data from proteomic analysis and the work of other research groups have provided the first answers. Trichoderma sp. is able to produce at least three different types of elicitors: elicitors made of enzymes or peptides, avr proteins, and oligosaccharides or lowmolecular-weight compounds released by the action of specific Trichoderma enzymes on fungal and plant cell walls.

The first type of elicitors may include different peptides and proteins, such as those reported by Hanson and Howell in Harman et al. (9), which have a size range from 6.2 to $42 \mathrm{kDa}$, possibly include a serine protease and a xylanase, and induce in the plant the biosynthesis of terpenoid phytoalexins and peroxidase. Based on yet unpublished data collected in our laboratory, we believe that among the Trichoderma elicitors there are some of the proteins that the fungus uses for its mycoparasitic and antagonistic activities, such as chitinases and glucanases. Our idea is that some of the enzymes are secreted by Trichoderma spp. at low levels to look for the pathogen, and then produced profusely when its presence is sensed, and these compounds can be recognized by the plant as foreign fungal molecules (regardless of their enzymatic 
activity) probably by using specific receptors and resistance genes, just like it occurs with pathogens. In other words, by sensing the Trichoderma metabolites, the plant may become aware of the "battle" eventually occurring in the rhizosphere and quickly activates the defense system before or immediately after the attack. Finally, this may improve the ability of the plant to recognize potential pathogens. Our hypothesis fits with the observation that the increased resistance effect mediated by Trichoderma sp. is based on the pre-activation of the plant antimicrobial system rather than on the over-accumulation of defense proteins.

The second type of elicitors may be both avr-like proteins similar to those found in avirulent pathogens and Trichoderma spp. own avr proteins. The presence of the first has been indicated recently, with homologues of the C. fulvum avr 9 and sequences hybridizing with an avr4 probe found in T. harzianum and T. atroviride (9; M. Ruocco and M. Lorito, unpublished data). The presence of Trichoderma-specific avr genes has also been investigated, with several putative proteins being isolated and tested recently in our laboratory. This is an attractive concept from a biotechnological point of view because $a v r$ genes from biocontrol fungi like Trichoderma spp., that are avirulent on a great variety of plants, may be useful to provide protection against a large spectrum of pathogens. This could be achieved by transforming the Trichoderma avr genes in plants, applying ectopically their products or using them to genetically improve beneficial microbes. In fact, if the corresponding resistance genes are widely spread in the plant kingdom, this strategy may overcome the limitation of the race-associated $a v r$ genes obtained from pathogens.

The third type of elicitors are molecules released from the pathogen and the plant cell wall by the Trichoderma CWDEs. These molecules stimulate the biocontrol fungus and its antagonistic activity by activating the mycoparasitic gene expression cascade, but also act as elicitors when plant cells are exposed to them or when injected under the root and leaf surface (S. L. Woo and M. Lorito, unpublished data). Some of the novel mycoparasitic-related inducers and plant elicitors have been purified and characterized and found to be short oligosaccharides made of two types of monomers, with and without an amino acid residue. This provides a novel possibility of using the enzymes secreted by Trichoderma, rather than the fungus itself, to induce ISR in the plant. These powerful enzyme mixtures can be cheaply produced in large quantities by growing the fungus on simple substrates, easily separated from the biomass and the low molecular weight fraction, dried and formulated for a spray or drench application. This treatment may at the same time stimulate the activity of resident or introduced Trichoderma strains and activate the ISR system by releasing inducers for the fungus and elicitors for the plant, in addition to their innate antimicrobial effect (16). The few experiments so far performed in different laboratories with crude enzyme preparations have reduced lesions caused by $B$. cinerea on stored apples, and by Colletotrichum spp. on strawberry apices growing in a greenhouse (M. Lorito, Y. Elad, and S. Freeman, unpublished data).

Finally, understanding the mechanism of interaction between Trichoderma spp. and the plant has provided for the first time the opportunity to genetically increase the ability of a $T$. atroviride strain to induce ISR. We were able to overexpress a glucose oxidase gene from Aspergillus niger in Trichoderma strain P1 (20) and obtain mutants that, in vitro and in soil experiments, performed much better than the wild type both as mycoparasites and ISR inducing agents (3). In this case, the mutant spores applied to bean seeds produced plants that were more resistant to leaf infection by $B$. cinerea compared with those treated with the wild type, mainly because the high glucose oxidase activity expressed by the genetically improved Trichoderma spp. catalyzed the production of hydrogen peroxide and reactive oxygen species that systemically alerted the plant defense mechanism.

\section{ACKNOWLEDGMENTS}

Work by the authors has been supported by the following projects: FIRB-MIUR 2002; PON-MIUR 2002; EU TRICHOEST; EU 2E-BCAs, EU FAIR 98PL-4140; MIUR-MIPAF 2003; MIUR PRIN 2004.

\section{LITERATURE CITED}

1. Baek, J. M., Howell, C. R., and Kenerley, C. M. 1999. The role of an extracellular chitinase from Trichoderma virens Gv29-8 in the biocontrol of Rhizoctonia solani. Curr. Genet. 35:41-50.

2. Benitez, T., Rincon, A. M., Limon, M. C., and Codon, A. C. 2004. Biocontrol mechanisms of Trichoderma strains. Int. Microbiol. 7:249-260.

3. Brunner, K., Zeilinger, S., Ciliento, R., Woo, S. L., Lorito, M., Kubicek, C. P., and Mach, R. L. 2005. Genetic improvement of a fungal biocontrol agent to enhance both antagonism and induction of plant systemic disease resistance. Appl. Environ. Microbiol. 71:3959-3965.

4. Carsolio, C., Benhamou, N., Haran, S., Cortes, C., Gutierrez, A., Chet, I., and Herrera-Estrella, A. 1999. Role of the Trichoderma harzianum endochitinase gene, ech42 in mycoparasitism. Appl. Environ. Microbiol. 65:929-935.

5. Ciliento, R., Donzelli, B., Woo, S. L., Del Sorbo, G., Harman, G. E., Scala, F., and Lorito, M. 2000. Cloning and disruption of a new beta-1,3glucosidase-encoding gene in Trichoderma harzianum. Page 84 in: Proc. 5 th Congress of the European Foundation for Plant Pathology, Taormina, Italy.

6. Fogliano, V., Ballio, A., Gallo, M., Woo, S. L., Scala, F., and Lorito, M. 2002. Pseudomonas lipodepsipeptides and fungal cell wall-degrading enzymes act synergistically in biological control. Mol. Plant-Microbe Interact. 15:323-333.

7. Grinyer, J., Hunt, S., McKay, M., Herbert, B. R., and Nevalainen, H. 2005. Proteomic response of the biological control fungus Trichoderma atroviride to growth on the cell walls of Rhizoctonia solani. Curr. Genet. 47:381-383.

8. Grinyer, J., McKay, M., Nevalainen, H., and Herbert, B. R. 2004. Fungal proteomics: Initial mapping of biological control strain Trichoderma harzianum. Curr. Genet. 45:163-169.

9. Harman, G. E., Howell, C. R., Viterbo, A., Chet, I., and Lorito, M. 2004. Trichoderma species-opportunistic, avirulent plant symbionts. Nature Rev. Microbiol. 2:43-56.

10. Harman, G. E., and Kubicek, C. P. 1998. Trichoderma and Gliocladium, Vol. 2. Enzymes, Biological Control and Commercial Applications. Taylor \& Francis, London.

11. Harman, G. E., Lorito, M., and Lynch, J. M. 2004. Uses of Trichoderma spp. to alleviate or remediate soil and water pollution. Adv. Appl. Microbiol. 56:313-330.

12. Kubicek, C. P., and Harman, G. E. 1998. Trichoderma and Gliocladium. Vol. 1. Basic Biology, Taxonomy and Genetics. Taylor \& Francis, London.

13. Kubicek, C. P., Mach, R. L., Peterbauer, C. K., and Lorito, M. 2001. Trichoderma: From genes to biocontrol. J. Plant Pathol. 83:11-23.

14. Lanzuise, S., Ruocco, M., Catapano, L., Scala, V., Ciliento, R., Ambrosino, P., Woo, S., Scala, F., Cecere, G., Del Sorbo, G., and Lorito, M. 2003. ABC transporter genes of Trichoderma spp., and their involvement in biocontrol. (Abstr.) J. Plant Pathol. 85:301.

15. Lim, D., Hains, P., Walsh, B., Bergquist, P., and Nevalainen, H. 2001. Proteins associated with the cell envelope of Trichoderma reesei: A proteomic approach. Proteomics 1:899-909.

16. Lorito, M., Harman, G. E., Hayes, C. K., Broadway, R. M., Tronsmo, A., Woo, S. L., and Di Pietro, A. 1993. Chitinolytic enzymes produced by Trichoderma harzianum: Antifungal activity of purified endochitinase and chitobiosidase. Phytopathology 83:302-307.

17. Lorito, M., Scala, F., Zoina, A., and Woo, S. L. 2001. Enhancing biocontrol of fungal pests by exploiting the Trichoderma genome. Pages 248-259 in: Enhancing Biocontrol Agents and Handling Risks. M. Vurro and J. Gressel, eds. IOS Press, Amsterdam.

18. Lorito, M., Woo, S. L., D’Ambrosio, M., Harman, G. E., Hayes, C. K., Kubicek, C. P., and Scala, F. 1996. Synergistic interaction between cell wall degrading enzymes and membrane affecting compounds. Mol. PlantMicrobe Interact. 9:206-213.

19. Lu, Z., Tombolini, R., Woo, S. L., Zeilinger, S., Lorito, M., and Jansson, J. K. 2004. In vivo study of Trichoderma-pathogen-plant interactions with constitutive and inducible GFP reporter systems. Appl. Environ. Microbiol. 70:3073-3081.

20. Mach, R. L., Peterbauer, C. K., Payer, K., Jaksits, S., Woo, S. L., Zeilinger, S., Kullnig, C. M., Lorito, M., and Kubicek, C. P. 1999. Expression of two major chitinase genes of Trichoderma atroviride ( $T$. harzianum P1) is triggered by different regulatory signals. Appl. Environ. Microbiol. 65:1858-1863. 
21. Monte, E. 2001. Understanding Trichoderma: Between biotechnology and microbial ecology. Int. Microbiol. 4:1-4.

22. Rey, M., Llobell, A., Monte, E., Scala, F., and Lorito, M. 2004. Genomics of Trichoderma. Pages 225-248 in: Applied Mycology and Biotechnology, vol. 4 Fungal Genomics. D. K. Arora and G. G. Khachatourians, eds. Elsevier B.V., Amsterdam, The Netherlands.

23. Woo, S. L., Donzelli, B., Scala, F., Mach, R., Harman, G. E., Kubicek, C. P., Del Sorbo, G., and Lorito, M. 1999. Disruption of the ech42 (endochitinase-encoding) gene affects biocontrol activity in Trichoderma harzianum P1. Mol. Plant-Microbe Interact. 12:419-429.

24. Woo, S. L., Fogliano, V., Mach, R. L., Scala, F., Zoina, A., Kubicek, C. P., and Lorito, M. 2002. Mycoparasitic Trichoderma strains are activated by host-derived molecules. Page 306 in: Proc. 6th European Conference on Fungal Genetics. G. Vannacci and S. Sarrocco, eds. Edizioni Plus, Pisa, Italy.

25. Woo, S. L., Ruocco, M., Ciliento, R., Lanzuise, S., Vinale, F., Formisano, E., Scala, V., Turrà, D., Scala, F, Zoina, A., Abadi, K., and Lorito, M. 2003. Molecular factors involved in the interaction between plants, pathogens and biocontrol fungi. Page 368 in: Proc. 11th International Congress on Molecular Plant-Microbe Interactions. St. Petersburg, Russia.
26. Yedidia, I., Benhamou, N., and Chet, I. 1999. Induction of defense responses in cucumber plants (Cucumis sativus L.) by the biocontrol agent Trichoderma harzianum. Appl. Environ. Microbiol. 65:10611070.

27. Yedidia, I., Benhamou, N., Kapulnik, Y., and Chet, I. 2000. Induction and accumulation of PR proteins activity during early stages of root colonization by the mycoparasite Trichoderma harzianum strain T-203. Plant Physiol. Biochem. 38:863-873.

28. Yedidia, I., Shoresh, M., Kerem, Z., Benhamou, N., Kapulnik, Y., and Chet, I. 2003. Concomitant induction of systemic resistance to Pseudomonas syringae pv. lachrymans in cucumber by Trichoderma asperellum (T-203) and accumulation of phytoalexins. Appl. Environ. Microbiol. 69:7343-7353.

29. Yedidia, I., Srivastva, A. K., Kapulnik, Y., and Chet, I. 2001. Effect of Trichoderma harzianum on microelement concentrations and increased growth of cucumber plants. Plant Soil 235:235-242.

30. Zeilinger, S., Galhaup, C., Payer, K., Woo, S. L., Mach, R. L., Fekete, C., Lorito, M., and Kubicek, C. P. 1999. Chitinase gene expression during mycoparasitic interaction of Trichoderma harzianum with its host. Fungal Genet. Biol. 26:131-140. 